\title{
A Case Report of Hepatocellular Carcinoma Derived from Rastelli Procedure-Related Congestive Liver Disease
}

\section{Yoshihiro Sakano}

Graduate school of medicine, Osaka university

\section{Takehiro Noda}

Graduate school of medicine, Osaka university

Shogo Kobayashi ( $\sim$ s-kobayashi@umin.ac.jp )

Graduate school of medicine, Osaka university

Yoshifumi Iwagami

Graduate school of medicine, Osaka university

Daisaku Yamada

Graduate school of medicine, Osaka university

Yoshito Tomimaru

Graduate school of medicine, Osaka university

Hirofumi Akita

Graduate school of medicine, Osaka university

Kunihito Gotoh

Graduate school of medicine, Osaka university

Koji Umeshita

Graduate school of medicine, Osaka university

\section{Yuichiro Doki}

Graduate school of medicine, Osaka university

Hidetoshi Eguchi

Graduate school of medicine, Osaka university

\section{Case report}

Keywords: congenital, resection, hepatectomy, liver cancer,

Posted Date: March 10th, 2021

DOI: https://doi.org/10.21203/rs.3.rs-280374/v1 
License: (c) (i) This work is licensed under a Creative Commons Attribution 4.0 International License. Read Full License 


\section{Abstract}

[Background] The prognosis of congenital heart disease in children has improved, but late complications in adulthood are becoming an important problem. One late complication after congenital heart surgery is congestive liver disease, leading to liver cirrhosis and hepatocellular carcinoma (HCC). The Rastelli procedure is one of the surgical methods for transposition of the great arteries, and patients are thought have a low post-surgical risk of congestive hepatopathy by receiving re-intervention for right ventricular outflow tract obstruction.

[Case presentation] We present the first case of HCC derived from Rastelli procedure-related congestive liver disease in a 41-year-old male. The patient underwent the Rastelli operation at 2 years of age and right ventricular outflow tract reconstruction at 10 and 35 years of age due to right ventricular outflow tract obstruction. At 41 years of age, a hepatic tumor was detected by computed tomography. Abdominal enhancing computed tomography revealed a partially hypervascular tumor in segment 2 in early phase and wash-out in late phase. The patient was diagnosed with HCC and underwent left lateral segmentectomy of the liver, splenectomy, and partial gastrectomy. The patient was discharged on the 28th postoperative day without postoperative complications. The resected tumor pathologically revealed moderately differentiated HCC and F3 liver fibrosis.

[Conclusions] In the management of patients after the Rastelli operation, surveillance for congestive liver disease and HCC development is important, even if the patients have undergone right ventricular outflow tract reconstruction.

\section{Background}

The reported prevalence of congenital heart disease (CHD) varies between 4 and 10 per 1000 live births [1]. The prognosis of CHD in children has improved greatly in recent years, as advances in congenital heart surgery and congenital cardiology have enabled more than $90 \%$ of cases to reach adulthood [2]. However, late complications after congenital heart surgery, such as arrhythmia, heart failure, heart valve disease, renal failure, and congestive liver disease, are becoming an important problem in these adult patients [3]. Congestive liver disease is an important complication because it often leads to liver cirrhosis, and some cases have demonstrated the occurrence of hepatocellular carcinoma (HCC). Chronic liver disease due to heart disease is called cardiac cirrhosis. Cardiac cirrhosis commonly occurs as a result of cardiac conditions that permit long-term survival with marked elevation of the systemic venous pressure [4]. The representative congestive liver disease after congenital heart surgery is Fontan-associated liver disease (FALD). The Fontan operation is applied in the case of a single ventricle and involves separation of the pulmonary circulation from the systemic circulation. The Fontan physiology after surgery can lead to elevated systemic venous pressure and diminished cardiac output, leading to liver fibrosis and HCC with the duration of the Fontan circulation [5], [6]. However, to the best of our knowledge, there have been no reports of HCC with congestive liver fibrosis after the Rastelli operation. 
The Rastelli procedure is one of the methods for treating transposition of the great arteries, which requires intraventricular rerouting from the right ventricle to the pulmonary artery [3]. Although early mortality and morbidity are low, many patients undergoing the Rastelli procedure eventually require reintervention for right ventricular outflow tract obstruction (RVOTO). The incidence of pulmonary artery stenosis requiring re-intervention has been reported to be approximately $20 \%$, and pulmonary artery angioplasty or stent replacement have been the most common procedures [7]' [8]. By undergoing these reinterventions, the patients have a lower risk of elevated systemic venous pressure and congestive hepatopathy than the patients who undergo the Fontan procedure.

Here, we describe the first case of HCC derived from Rastelli procedure-related congestive liver disease in a 41 -year-old male patient.

\section{Case Presentation}

The patient was a 41-year-old male diagnosed with truncus arteriosus shortly after birth. The patient underwent the Rastelli procedure at 2 years of age. The systemic venous pressure before and after the Rastelli procedure was 11 and $8 \mathrm{mmHg}$, respectively. When the patient was 10 years old, he was diagnosed with RVOTO and underwent right ventricular outflow tract reconstruction. He underwent the procedure again at 35 years old. The systemic venous pressure was approximately $12 \mathrm{mmHg}$ before each reconstruction. After the last surgery, he presented no clinical symptoms of chronic heart failure. At 41 years old, a hepatic tumor suspected of HCC was detected by computerized tomography (CT). The patient was referred to our hospital for treatment of the hepatic tumor. His laboratory tests showed elevated levels of $\mathrm{y}$-glutamyl transpeptidase $(130 \mathrm{U} / \mathrm{L})$, a-fetoprotein $(2488 \mathrm{ng} / \mathrm{ml})$, and des-ccarboxyprothrombin $(24321 \mathrm{mAU} / \mathrm{mL})$. We found no evidence of viral hepatitis, steatohepatitis, autoimmune liver disease, or metabolic liver disease. Abdominal CT demonstrated a tumor $7 \mathrm{~cm}$ in diameter in the left lobe of the liver with low intensity, which was partially enhanced in early phase and showed wash-out in late phase (Fig. 1a-1b). The placed pacemaker was not compatible with magnetic resonance imaging. Positron emission tomography/CT with 18-fluorodeoxyglucose (FDG) revealed FDG uptake in the tumor (Fig. 1C). Therefore, the patient was diagnosed with HCC. Cardiac catheterization showed that his cardiac index $\left(3.7 \mathrm{~L} /\right.$ minute $\left./ \mathrm{m}^{2}\right)$ and pulmonary artery wedge pressure $(17 \mathrm{mmHg})$ were maintained, but the systemic venous pressure was slightly elevated to $16 \mathrm{mmHg}$.

After the catheterization, he had acute abdominal pain and HCC rupture was shown. The patient underwent emergency transcatheter arterial embolization (TAE) to stop the bleeding. Twenty days after $T A E$, the patient underwent hepatic resection. The tumor adhered to the stomach and spleen because of inflammation after TAE; therefore, hepatic left lateral segmentectomy, splenectomy, and partial gastrectomy was performed. The intraoperative central venous pressures shifted $9-23 \mathrm{mmHg}$. The operation time was 546 minutes and blood loss $3350 \mathrm{ml}$. The patient was discharged on the 28th postoperative day without postoperative complications. 
The resected tumor exhibited expansive and extrahepatic growth with extracapsular invasions. Splenomegaly was observed (Fig. 2a). The tumor cells were polymorphic with an increased nuclear/cytoplasmic ratio and chromatin-rich nuclei, and the pathological diagnosis was moderately and poorly differentiated HCC. Intratumoral bleeding and partial necrosis by TAE were shown (Fig. 2b). The noncancerous area of the resected specimen revealed bridging fibrosis without fat deposition. Perisinusoidal and peri-portal fibrosis were observed, and fibrosis around the central vein was dominant. The inflammation activity was moderate (A2) using the hematoxylin and eosin stain. Azan staining showed clear bridging fibrosis, and the stage of fibrosis was diagnosed as F3 (Fig. 2c-2d).

Figure 2 Macroscopic and microscopic findings in the resected tissue specimen. (a) The resected tumor showed expansive and extrahepatic growth. (b) Pathological examination revealed that the tumor was moderately and poorly differentiated HCC. Intratumoral bleeding and partial necrosis were found. (c) The noncancerous lesions exhibited peri-sinusoidal and peri-portal fibrosis. The inflammation activity was moderate. (d) Azan staining showed clear bridging fibrosis, and the stage of fibrosis was diagnosed as F3. Scale bar, $500 \mu \mathrm{m}(\mathrm{b}-\mathrm{d}) .</$ fig $>$

\section{Discussion}

Congestive liver disease includes a wide range of structural and functional disorders of the liver secondary to chronic liver congestion caused by elevated systemic venous pressure and/or low cardiac output of new circulation after congenital heart surgery. The spectrum of congestive liver diseases ranges from mild hepatic congestion to liver cirrhosis and may lead to the complications of portal hypertension and HCC [9]. In patients with congestive liver disease, the pathophysiology is quite different from the inflammatory mechanisms of toxic products, such as alcohol or drugs, and viral infections, such as hepatitis $B$ or $C$ virus [10]. An elevated central venous pressure is easily transmitted to the liver because of its location. If this pressure is maintained, it causes congestion and increased intrahepatic venous pressure. Microscopically, these phenomena result in sinusoidal dilatation, which is more evident in the centrilobular area and fibrosis around the central vein. In our case, pathological findings revealed F3 liver fibrosis around the central vein areas and chronic hepatitis according to congestion.

The most famous congestive liver disease is FALD. In addition to chronic inflammation caused by longlasting liver congestion, $\mathrm{HCC}$ is one of the most serious complications. As cardiologists are becoming familiar with FALD, more patients are undergoing regular abdominal ultrasonography or CT after the Fontan operation for surveillance. The annual risk of HCC in FALD-associated cirrhosis is comparable to that of cirrhosis of other etiology, 1.5-5\% [11]. The American Association for the Study of Liver Diseases guidelines recommend that cirrhotic patients be enrolled in HCC surveillance with 6-month abdominal ultrasonography [12]. Thus, surveillance for HCC is warranted in patients at risk of congestive liver diseases, such as those who have undergone the Fontan operation.

In 1968, Rastelli conceived a new technique for repairing transposition of the great arteries in the presence of a ventricular septal defect based on redirection of the ventricular outflows. This technique 
was named the Rastelli procedure and has become the standard surgical procedure for patients with transposition of the great arteries. Despite good early results, the Rastelli procedure has been reported to have disappointing results regarding late morbidity and mortality. According to a recent study, the mortality associated with the Rastelli procedure has improved, with a more than $90 \%$ survival rate at 20 years. However, almost half of patients need to undergo reoperation for RVOTO.[13] Mechanisms leading to RVOTO include conduit stenosis and patient's somatic growth [14] [15]. Thus, most patients need to have the reoperation to prevent heart failure [16]. Reoperation in patients after the Rastelli procedure could control the condition of congestive liver diseases, and the risk of developing congestive liver cirrhosis and HCC is considered to be low. However, our case showed F3 liver fibrosis and liver cancer carcinogenesis despite two ventricular outflow tract reconstructions. Our case indicates the importance of surveillance for detecting liver cirrhosis and HCC by ultrasound or CT.

\section{Conclusion}

In conclusion, we report the first case of an adult patient developing HCC derived from Rastelli procedurerelated congestive liver disease. In the management of patients after the Rastelli operation, surveillance for congestive liver disease and HCC development is important even if the patients have undergone right ventricular outflow tract reconstruction.

\section{Declarations}

\section{Ethics approval and consent to participate}

All procedures used in this research were approved by the Ethical Committee of

Graduate School of Medicine Osaka University(No.15145-2).

\section{Consent for publication}

Written informed consent was obtained from the patients for publication of this case report and any accompanying images. A copy of the written consent is available for review by the Editor-in-Chief of this journal.

\section{Availability of data and materials}

Data sharing is not applicable to this article as no datasets were generated or analyzed during the current study.

\section{Competing interests}

The authors declare that they have no competing interests

\section{Funding}


This work was not supported.

\section{Authors' contributions}

Yoshihiro Sakano and Takehiro Noda treated the patient and conceived the idea. Yoshihiro Sakano performed the literature search and wrote the manuscript. Yoshihiro Sakano and Takehiro Noda reviewed and revised manuscript. All authors have read and approved the final manuscript.

\section{Acknowledgements}

We greatly appreciate the assistance of the staff of the Department of Gastroenterological Surgery, Graduate School of Medicine, Osaka University, and thank them for their efforts.

\section{References}

1. Helm PC, Kempert S, Körten MA, Lesch W, Specht K, Bauer UMM. Congenital heart disease patients' and parents' perception of disease-specific knowledge: Health and impairments in everyday life. Congenit Heart Dis. 2018;13:377-83.

2. Warnes CA, Williams RG, Bashore TM, Child JS, Connolly HM, Dearani JA, del Nido P, Fasules JW, Graham TP Jr, Hijazi ZM, et al. ACC/AHA 2008 Guidelines for the Management of Adults with Congenital Heart Disease: a report of the American College of Cardiology/American Heart Association Task Force on Practice Guidelines (writing committee to develop guidelines on the management of adults with congenital heart disease). Circulation. 2008;118:e714-833.

3. Toyoda Y, Hiramatsu T, Nagashima M, Matsumura G, Yamazaki K. Long-term Effect of Enlargement of a Ventricular Septal Defect in the Rastelli Procedure. Semin Thorac Cardiovasc Surg. 2017;29:215-20.

4. Angelico R, Lisignoli V, Monti L, Pariante R, Grimaldi C, Saffioti MC, Gagliardi MG, Spada M. Laparoscopic liver resection for hepatocellular carcinoma in Fontan-associated chronic liver disease. The first case report. Int J Surg Case Rep. 2019;59:144-7.

5. Téllez L, Rodríguez de Santiago E, Albillos A. Fontan-associated Liver Disease. Rev Esp Cardiol (Engl Ed). 2018;71:192-202.

6. Yokota Y, Noda T, Kobayashi S, Matsumoto K, Sakano Y, Iwagami Y, Tomimaru Y, Akita H, Gotoh K, Umeshita K, et al. A case report of Fontan procedure-related hepatocellular carcinoma: pure laparoscopic approach by low and stable pneumoperitoneum. BMC Surg. 2020;20:80.

7. Lee J, Abdullah Shahbah D, El-Said H, Rios R, Ratnayaka K, Moore J. Pulmonary artery interventions after the arterial switch operation: Unique and significant risks. Congenit Heart Dis. 2019;14:288-96.

8. Lalezari S, Bruggemans EF, Blom NA, Hazekamp MG. Thirty-year experience with the arterial switch operation. Ann Thorac Surg. 2011;92:973-9.

9. Yoon JS, Lee DH, Cho EJ, Song MK, Choi YH, Kim GB, Lee YB, Lee JH, Yu SJ, Kim H, et al: Risk of Liver Cirrhosis and Hepatocellular Carcinoma after Fontan Operation: A Need for Surveillance. 
Cancers (Basel) 2020, 12.

10. Fernández-Iglesias A, Gracia-Sancho J. How to Face Chronic Liver Disease: The Sinusoidal Perspective. Front Med (Lausanne). 2017;4:7.

11. Asrani SK, Warnes CA, Kamath PS. Hepatocellular carcinoma after the Fontan procedure. N Engl J Med. 2013;368:1756-7.

12. Heimbach JK, Kulik LM, Finn RS, Sirlin CB, Abecassis MM, Roberts LR, Zhu AX, Murad MH, Marrero JA. AASLD guidelines for the treatment of hepatocellular carcinoma. Hepatology. 2018;67:358-80.

13. Brown JW, Ruzmetov M, Huynh D, Rodefeld MD, Turrentine MW, Fiore AC. Rastelli operation for transposition of the great arteries with ventricular septal defect and pulmonary stenosis. Ann Thorac Surg. 2011;91:188-93. discussion 193 - 184.

14. Kreutzer C, De Vive J, Oppido G, Kreutzer J, Gauvreau K, Freed M, Mayer JE Jr, Jonas R, del Nido PJ. Twenty-five-year experience with rastelli repair for transposition of the great arteries. $J$ Thorac Cardiovasc Surg. 2000;120:211-23.

15. Al-Jughiman MK, Al-Omair MA, Van Arsdell GS, Morell VO, Jacobs ML. D-Transposition of the Great Arteries with Ventricular Septal Defect and Left Ventricular Outflow Tract Obstruction (DTGA/VSD/LVOTO): A Survey of Perceptions, Preferences, and Experience. Pediatr Cardiol. 2015;36:896-905.

16. Cleuziou J, Vitanova K, Pabst von Ohain J, Ono M, Tanase D, Burri M, Lange R. Incidence and Risk Factors for Right Ventricular Outflow Tract Obstruction after the Arterial Switch Operation. Thorac Cardiovasc Surg. 2019;67:37-43.

\section{Figures}



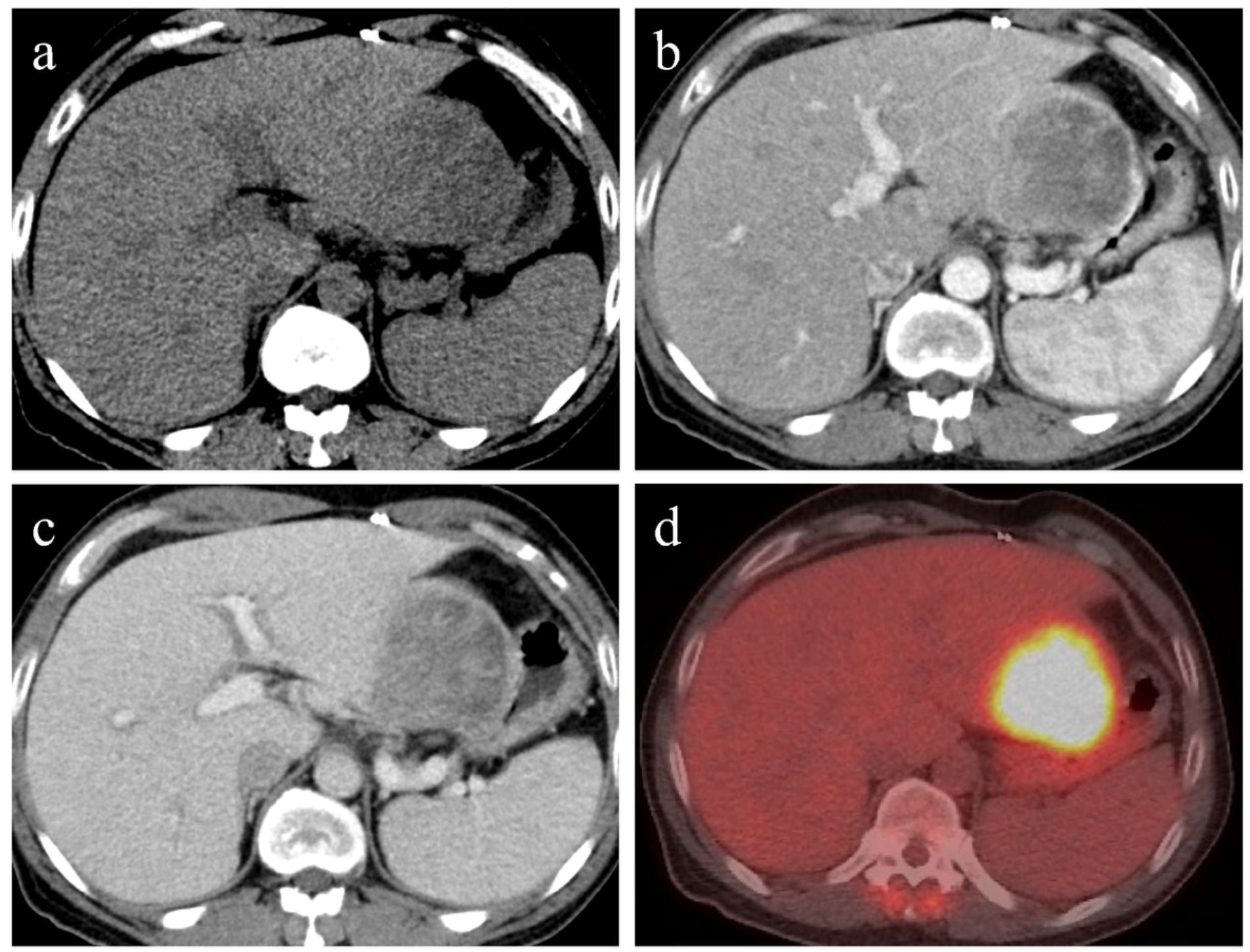

\section{Figure 1}

Abdominal contrast-enhanced CT and positron emission tomography/CT with FDG. (a) Abdominal CT revealed a low density mass in the left lobe of the liver. (b, c) Enhancing agent showed a partially hypervascular mass in the left lobe in arterial phase and wash-out in late phase. (d) Positron emission tomography/CT showed increased FDG uptake in the hepatic lesion. 

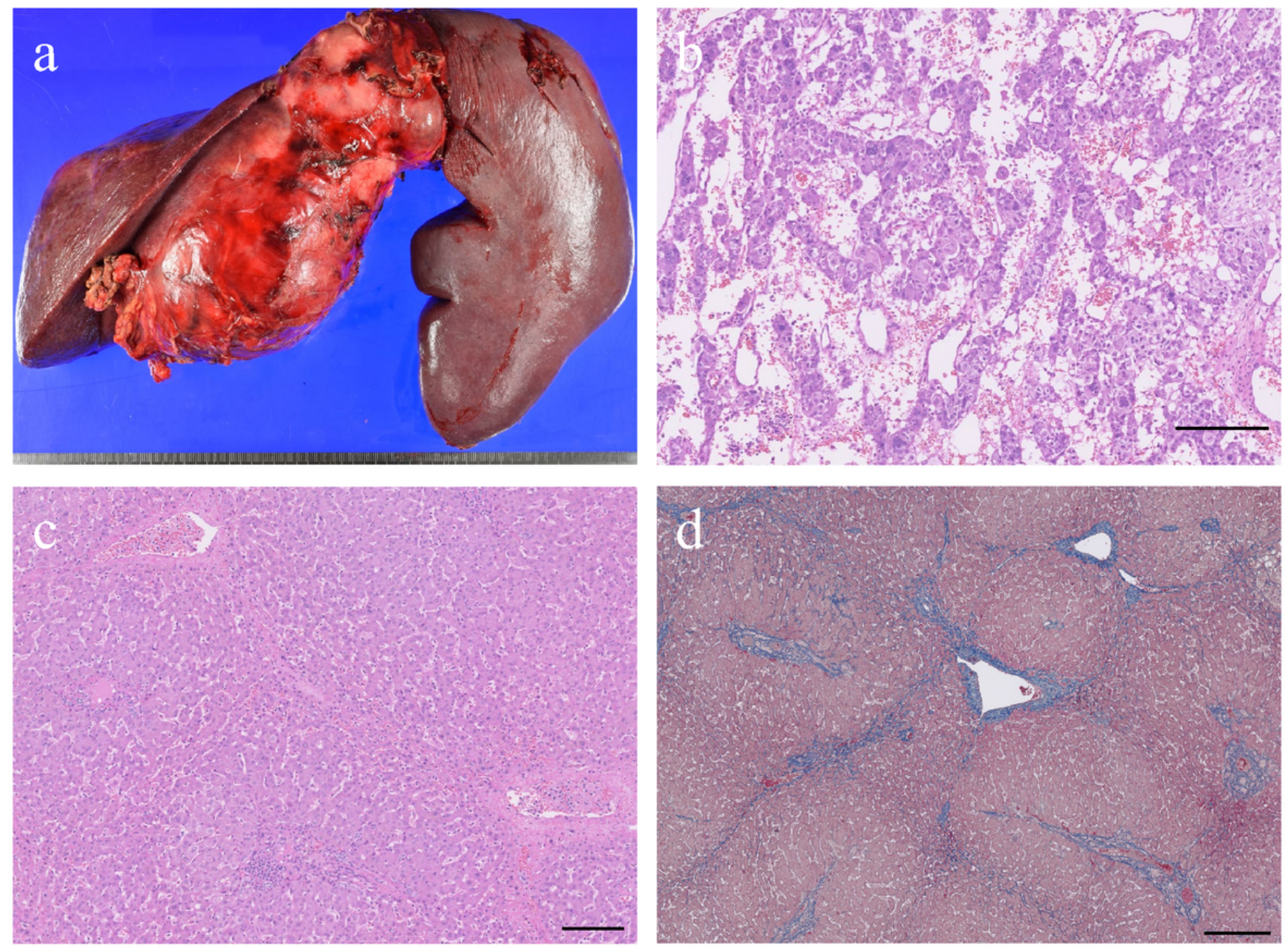

\section{Figure 2}

Macroscopic and microscopic findings in the resected tissue specimen. (a) The resected tumor showed expansive and extrahepatic growth. (b) Pathological examination revealed that the tumor was moderately and poorly differentiated HCC. Intratumoral bleeding and partial necrosis were found. (c) The noncancerous lesions exhibited peri-sinusoidal and peri-portal fibrosis. The inflammation activity was moderate. (d) Azan staining showed clear bridging fibrosis, and the stage of fibrosis was diagnosed as F3. Scale bar, $500 \mu \mathrm{m}(\mathrm{b}-\mathrm{d})$. 